\title{
mTOR-driven widespread exon skipping renders multifaceted gene regulation and proteome complexity
}

Sze Cheng ${ }^{1 \#}$, Naima Ahmed Fahmi ${ }^{2 \#}$, Meeyeon Park ${ }^{1}$, Jae-Woong Chang ${ }^{1}$, Jiao Sun ${ }^{2}$, Kaitlyn Thao $^{1}$, Hsin-Sung Yeh ${ }^{1}$, Wei Zhang ${ }^{2, *}$, and Jeongsik Yong,**

${ }^{1}$ Department of Biochemistry, Molecular Biology and Biophysics

University of Minnesota Twin Cities, Minneapolis, MN 55455; USA

${ }^{2}$ Department of Computer Science

University of Central Florida, Orlando, FL 32816; USA

\#These authors equally contributed to this work.

*Co-corresponding authors

Corresponding author e-mail: jyong@umn.edu,wzhang.cs@ucf.edu

Phone: 612-626-2420 (Jeongsik Yong) and 407-823-2763 (Wei Zhang)

Running Title: mTOR-Driven AS Multifariously Regulates Proteome

Keywords: mTOR, alternative splicing, post-transcriptional gene regulation, functional proteome 


\begin{abstract}
The mammalian target of rapamycin (mTOR) pathway is crucial in cell proliferation. Previously, we reported transcriptome-wide 3'-untranslated region (UTR) shortening by alternative polyadenylation upon mTOR activation and its impact on the proteome. Here, we further interrogated the mTOR-activated transcriptome and found that hyperactivation of mTOR promotes transcriptome-wide exon-skipping/exclusion, producing short isoform transcripts from genes. This widespread exon skipping confers multifarious regulations in the mTOR-controlled functional proteomics: alternative splicing (AS) in the 5'-UTR controls translation efficiency while AS in coding regions widely affects the protein length and functional domains. They also alter the half-life of proteins and affect the regulatory post-translational modifications. Among the RNA processing factors differentially regulated by mTOR signaling, we found that SRSF3 mechanistically facilitates exon skipping in the mTOR-activated transcriptome. This study reveals a role of $\mathrm{mTOR}$ in AS regulation and demonstrates that widespread AS is a multifaceted modulator of the mTOR-regulated functional proteome.
\end{abstract}




\section{Introduction}

mRNA splicing is a critical co-transcriptional process to produce uninterrupted coding DNA sequences (CDS) for protein translation ${ }^{1}$. Alternative splicing (AS) can occur by alternating the inclusion of exons or part of exons to produce mature mRNAs ${ }^{2-4}$. Thus, AS diversifies the proteome by potentiating the production of multiple protein isoforms from a gene ${ }^{5,6}$. Although AS serves as an essential layer of post-transcriptional regulation of gene expression in eukaryotes ${ }^{7,8}$, much remains to be explored concerning its regulation and impact on the resulting proteome.

Mammalian target of rapamycin (mTOR) is a serine/threonine kinase and is the major component of the mTOR Complex 1 (mTORC1) and 2 (mTORC2) $)^{9-11}$. Heterodimeric tuberous sclerosis complex composed of TSC1 and TSC2 is a negative regulator of mTORC1. mTOR promotes the translation of a subset of mRNAs that contain the 5'-terminal oligopyrimidine tract $\left(5^{\prime}-\mathrm{TOP}\right)^{12-15}$. mTOR was also shown to regulate select RNA-binding proteins (RBPs) and functions in post-transcriptional regulation ${ }^{16-18}$. Hyperactivation of mTOR leads to transcriptomewide alternative polyadenylation (APA) in the 3'-untranslated regions (3'-UTR) of transcripts and affects diverse cellular pathways ${ }^{19,20}$. mTOR activation also prefers the expression of splicing factor U2AF1a isoform to that of the $\mathrm{b}$ isoform and renders U2AF1a-dependent AS events ${ }^{21}$.

In this study, we investigated mTOR-driven transcriptome changes in alternative splicing and their impact on the resulting proteome using various cellular models. We found that widespread exon skipping by mTOR activation diversifies the proteome by changing functional domains, post-translational modifications, and protein stabilities. 


\section{Results and Discussion}

\section{mTOR activation leads to transcriptome-wide exon skipping}

To better understand mTOR-driven transcriptomic features, we profiled the transcriptome of various mammalian cells with low and high mTOR contents: $T s c 1^{-/}$mouse embryonic fibroblasts (MEFs), MCF7 and MDA-MB-361 cells treated with DMSO (mock) or Torin 1, a potent inhibitor of mTOR. From our initial interrogation that focused on the RNA processing

pathway, we found numerous RBPs

involved in AS and APA to be

differentially expressed, suggesting

the comprehensive role of mTOR in

the transcriptome (Fig. 1A and Suppl

Table 1). We then profiled AS events

using the custom-developed AS-

Quant pipeline ${ }^{22}$. Strikingly, we found

that chemical inhibition of mTOR in

$\mathrm{Tscl}^{-/-}$MEFs drives exon inclusion in

general: among the affected AS

events, exon inclusion in cassette type

AS was almost exclusively preferred

(497 events; 96\%) in Torin 1-treated

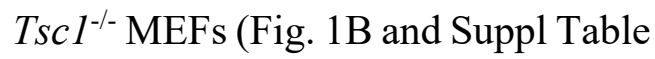

2). This trend of exon inclusion upon

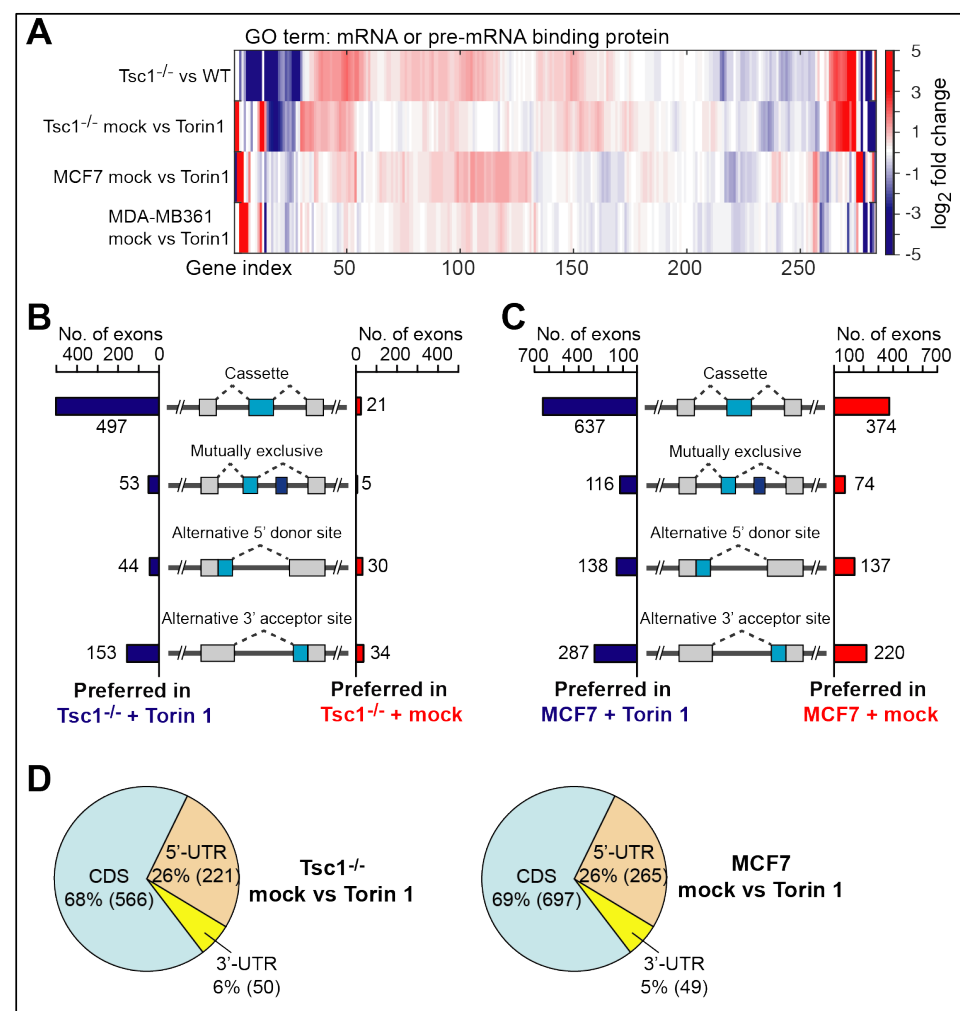

Figure 1. The mTOR-activated transcriptome features widespread exon skipping. A) Hierarchical clustering of select RNA-binding proteins (RBPs) using differential gene expression analysis in low and high mTOR cellular environments. Comparisons were made in the combination of cells as indicated. The $\mathrm{x}$-axis indicates the 285 different RBPs analyzed. The y-axis shows the combination of comparisons. The expression level is color-coded as shown in the scale bar and represents the $\log 2$ fold changes of gene expression. B-C) Analyses of four different types of alternative splicing events in B) $T s \mathrm{I}^{-/-}$mock vs. Torin 1 treatment and C) Breast cancer cell line MCF7 mock vs. Torin 1 treatment. Cases of alternative exon inclusion are compared and illustrated in the combination as indicated in the figure. D) Distribution of mTOR-regulated alternative splicing (AS) events in different regions of the mRNA. 
mTOR inhibition was consistent with other types of AS events with varying degrees (59\% in 5'AS site and 82\% in 3'-AS site) (Fig. 1B, Suppl Table 2). Consistent with these findings,

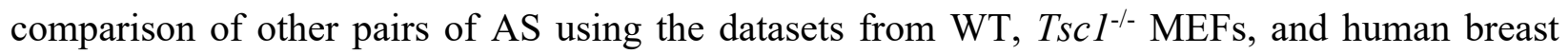
cancer cell lines MCF7 and MDA-MB-361 showed an overall increase of exon inclusion upon mTOR inhibition. (Fig. 1C, Suppl Fig. 1A-B, and Suppl Table 3-4). We noticed that the number of exon inclusions varies among profiled cellular models $(60 \%$ in breast cancer transcriptome compared to $90 \%$ in $\mathrm{Tscl}^{-/-}$MEF transcriptome). This discrepancy may come from the degree of mTOR activation in these cell lines examined: $T s c 1^{-/-}$MEFs are genetically programmed to hyperactivate mTOR signaling while MCF7 and MDA-MB-361 cells may have a reduced level of mTOR hyperactivation. Thus, upon mTOR inhibition, changes in AS events are maximized in the $T s c 1^{-/-}$system compared to breast cancer cells. In a similar context, when the same $T s c 1^{-/-}$ transcriptome was analyzed against the WT (basal mTOR activity) transcriptome, it showed less exon inclusion-biased AS events compared to that of the $T s c 1^{-/-}$transcriptome with Torin 1 treatment. We further delved into the nucleotide contexts surrounding the 3'-splice site and found that $\mathrm{mTOR}$ activation prefers a longer stretch of poly-pyrimidine tract ( -3 to -20 position) in the cassette exon and a weak poly-pyrimidine tract with biased $\mathrm{C}$ at the -1 position in the alternative 3'-splice site in both $T s c 1^{-/-}$MEFs and human cancer cells (Suppl Fig. 1C). Further examinations of affected AS events showed that the majority of them occur in the CDS of mRNA (65-68\%) while $26-27 \%$ of them are found in the 5'-UTR (Fig. 1D and Suppl Fig. 1D). These findings suggest that mTOR activation escalates the frequency of exon skipping during pre-mRNA splicing leading to preferential expression of short isoforms in the transcriptome.

\section{mTOR regulates translation through 5'-UTR AS}


Next, we validated mTOR-regulated AS events on select genes using PCR and analytical gel electrophoresis. Consistently, all tested genes showed exon skipping upon mTOR activation $\left(T s c 1^{-/-}\right.$MEFs mock vs Torin 1 treatment and $T s c 1^{-/-}$vs WT MEFs) (Fig. 2A, Suppl Fig. 2A). Interestingly, we found that a number of AS events in CDS where the exon-inclusion transcript contains a premature termination codon (PTC). Conversely, we also found cases where exoninclusion converts a PTC-containing isoform to an isoform with a full open reading frame. (Fig. 2B, Suppl Fig. 2B and C). In $M d m 4$, whose expression is important for the stability of p53 ${ }^{23}$, mTOR activation leads to the expression of evolutionary conserved PTC-containing Mdm4 transcript (NR_126506) by skipping exon 6 while mTOR inhibition switches back to the expression of full open reading frame (ORF) (NM_001302803) (Fig. 2B). Importantly, the PTCcontaining Mdm4 transcript degrades faster than the coding transcript (Fig. 2C and Suppl Fig. 2D). This suggests that mTOR-driven AS switches on and off gene expression by introducing PTC in select genes (Suppl Fig. 2B).

Besides the 5'-TOP feature of mRNAs ${ }^{24,25}$, the 5'-UTR length plays a role in mTORregulated translation ${ }^{26,27}$. Interrogation of 5'-UTR sequences found that the majority of genes showing mTOR-driven 5'-UTR AS do not contain the 5'-TOP motif (Fig. 2D). To examine the functional relevance of mTOR-modulated 5'-UTR length regulation in translation, two 5'-UTR AS isoforms lacking the 5'-TOP motif were analyzed in polysome profiling using analytical gel electrophoresis and cumulative plots (Fig. 2E and Suppl Fig. 2E-H). Cumulative plots of polysome profiling could determine statistically significant changes in the distribution of transcripts as exemplified by 5'-TOP containing transcripts Eef2 and Hsp90ab1 (Suppl Fig. 2G). All tested genes showed a difference in the polysome formation by the 5'-UTR AS: a short isoform of Gabpb2 formed polysome more efficiently and sustained polysome formation more rigorously upon Torin 





the characteristics of Gabpb2 short isoform in the polysome formation upon mock and Torin 1 treatment (Fig. 2E). The number of upstream open-reading-frames (uORFs) plays a critical role in determining the translation efficiency, and it has been shown to positively correlate with the length of the $5^{\prime}$-UTR ${ }^{28,29}$. However, our studies suggest that there is no unanimous theme of long vs. short in the formation or dissociation of polysome. This could be explained by exons being relatively short in length and AS may not be able to substantially modify the number of uORFs in the 5'UTR. Overall, these results suggest that 5'-UTR AS is a previously unrecognized element in translational regulation by $\mathrm{mTOR}$ and propose that it could be a resistance mechanism to mTOR inhibitors.

\section{AS contributes to the mTOR-programmed functional proteome at multiple levels}

To understand the functional relevance of mTOR-regulated AS in CDS, we first linked the affected exons to Pfam domains and used them for pathway analyses. From this we found 114 affected Pfam domains that are associated with various KEGG pathways and GO terms (Fig. 3A; Suppl Fig. 3A-B). AS of Sirt2 and Mdm2 in the CDS causes a frameshift in the original ORF and enforces the use of the second most available methionine for translation initiation. These events produce N-terminal truncated protein isoforms as illustrated in Fig. 3B and Suppl Fig. 3C. Cycloheximide chase assays on Flag-tagged SIRT2 and MDM2 isoforms showed that the Nterminal truncated short isoform of these proteins is less stable than the long isoform, suggesting that AS in CDS not only affects the functional domains but also changes the protein stability. We next asked whether mTOR-driven AS in CDS also affects proteome-wide post-translational modifications (PTMs) regulated by mTOR signaling ${ }^{30-33}$. We catalogued unique peptide 


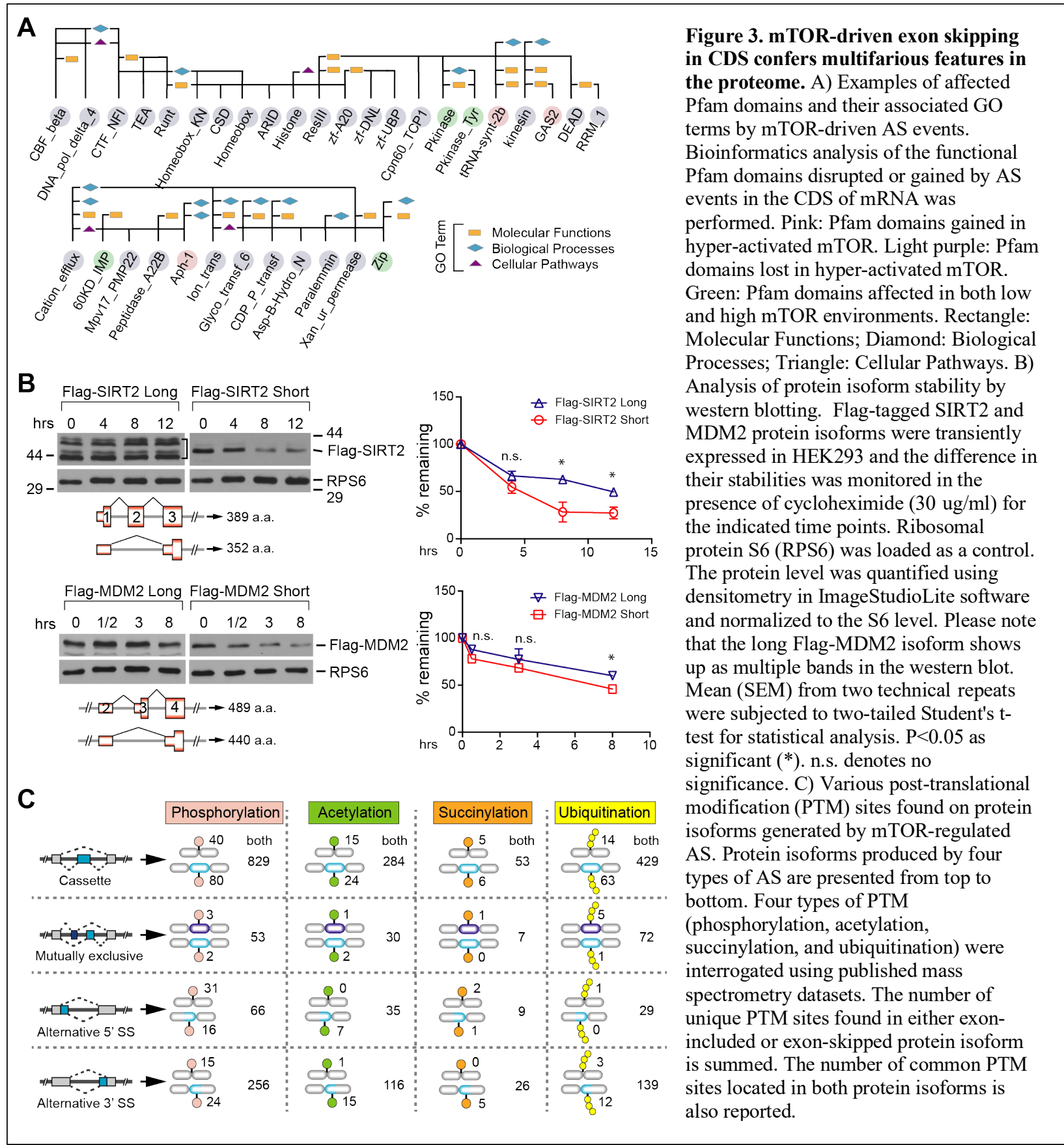

sequences belonging to each AS isoform and compared them to peptide sequences in published

PTM proteomic. In this proteome-wide search, we found that exon skipping could abolish existing sites for four interrogated PTMs in select genes and could also create new PTM sites in other genes, demonstrating that mTOR-modulated AS serves as a molecular scaffold for PTMs in functional proteomics (Fig. 3C). For example, the long SIRT2 isoform (NM_022432) creates a 
unique phosphoserine site that allows for cell cycle-dependent chromatin localization while the short isoform constitutively localizes to the cytoplasm ${ }^{34,35}$.

\section{SRSF3 promotes exon skipping in the mTOR-activated transcriptome}

Previously we reported the upregulation of splicing factor SRSF3 by mTOR-driven 3'UTR APA and suggested a suppressive role of SRSF3 in mutually exclusive $\mathrm{AS}^{36}$. To more broadly understand the effect of mTOR-activated SRSF3 upregulation on transcriptome-wide AS, we knocked down $\operatorname{Srsf3}$ expression in $T s c 1^{-/-}$MEFs and profiled AS events by RNA-Seq experiments (Fig. 4A). Interestingly, we found that $60 \%$ of cassette exon-inclusion cases are preferred in the $\operatorname{Srsf3}$ knockdown cells, suggesting that SRSF3 promotes exon-skipping. Further analysis of overlapping exon skipping events by SRSF3 and mTOR activation in $\mathrm{TSCl}^{-/-}$ MEFs showed that there is a $12.9 \%$ (64/497 cases) overlap (Fig. 4B), suggesting that SRSF3 upregulation is responsible for mTORdependent exon skipping. A similar observation was made when the $\operatorname{Srsf3}$ knockdown data were compared with the dataset of WT versus $T s c 1^{-/-}$MEFs

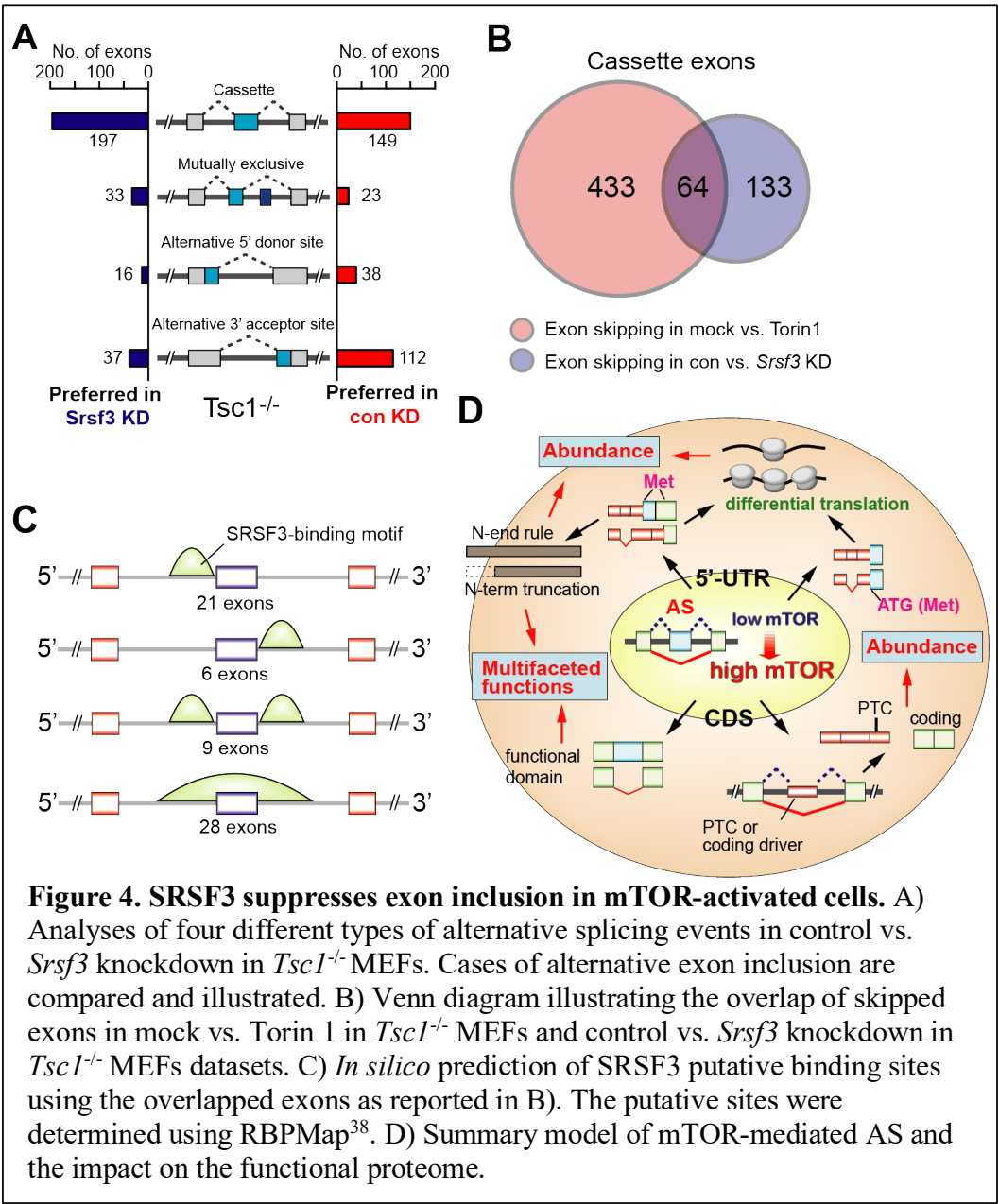


(16.1\%) (Suppl Fig. 4A). This suppressive role of SRSF3 on select genes was validated using RTPCR (Suppl Fig. 4B). To gain an insight into the suppression mechanism, an in silico search was conducted to map the consensus binding sequence of SRSF3 across the suppressed exon regions. This approach identified two major patterns of SRSF3-binding: a cluster of SRSF3 consensus motifs located immediately upstream of the 3'-splice site and nearly the entire suppressed exon regions (Fig. 4C). Previous transcriptome-wide footprinting for SRSF3 showed that SRSF3 binds to exon regions and prefers penta-pyrimidine sequences ${ }^{37}$. Thus, mTOR activation-dependent SRSF3 upregulation could suppress exon inclusion by competing for polypyrimidine tracts located upstream of the 3'-splice site. It could also compete with splicing enhancers to bind exons and suppress exon inclusion in AS.

This study identified mTOR-promoted transcriptome-wide exon skipping. These global AS events regulate the proteome multifariously: AS in CDS renders functional diversity and abundance of protein while AS in 5'-UTR mostly controls protein abundance (Fig. 4D). Further studies will be needed to understand the physiological relevance of AS in mTOR biology. Our study also identified SRSF3 as a key RNA-processing protein involved in mTOR-activated widespread exon skipping. Additional mechanistic experiments will be needed to further delineate other RBPs responsible for mTOR-regulated AS. 


\section{Acknowledgements}

We would like to thank Dr. Kwiatkowski at Harvard University for providing us $T s c 1^{-/-}$MEFs and matching wild type MEFs. We would also like to thank Dr. Yue Chen for providing us the lysine acetylation and succinylation proteomics datasets. We would also like to acknowledge Dr. Ann Hertzel for critically reading the manuscript and providing feedback. 
bioRxiv preprint doi: https://doi.org/10.1101/2020.02.27.967737; this version posted June 29, 2020. The copyright holder for this preprint (which was not certified by peer review) is the author/funder. All rights reserved. No reuse allowed without permission.

\section{Funding}

This work was supported by National Science Foundation [NSF-III1755761] to WZ and National Institutes of Health [1R01GM113952-01A1] and Department of Defense Congressionally Directed Medical Research Programs [W81XWH-16-1-0135] to JY. 
bioRxiv preprint doi: https://doi.org/10.1101/2020.02.27.967737; this version posted June 29, 2020. The copyright holder for this preprint (which was not certified by peer review) is the author/funder. All rights reserved. No reuse allowed without permission.

\section{Declarations}

The authors declare no conflict of interest. 


\section{References}

1. Han, J., Xiong, J., Wang, D. \& Fu, X. D. Pre-mRNA splicing: Where and when in the nucleus. Trends in Cell Biology 21, 336-343 (2011).

2. Faustino, N. A. \& Cooper, T. A. Pre-mRNA splicing and human disease. Genes and Development 17, 419-437 (2003).

3. Green, M. R. PRE-mRNA Splicing. Annu. Rev. Genet. 20, 671-708 (1986).

4. Jurica, M. S. \& Moore, M. J. Pre-mRNA splicing: Awash in a sea of proteins. Molecular Cell 12, 5-14 (2003).

5. Black, D. L. Protein diversity from alternative splicing: A challenge for bioinformatics and post-genome biology. Cell 103, 367-370 (2000).

6. Lopez, A. J. ALTERNATIVE SPLICING OF PRE-mRNA: Developmental Consequences and Mechanisms of Regulation. Annu. Rev. Genet. 32, 279-305 (1998).

7. Gonçalves, V., Pereira, J. F. S. \& Jordan, P. Signaling pathways driving aberrant splicing in cancer cells. Genes 9, (2018).

8. Lynch, K. W. Regulation of alternative splicing by signal transduction pathways. Advances in experimental medicine and biology 623, 161-174 (2007).

9. Saxton, R. A. \& Sabatini, D. M. mTOR Signaling in Growth, Metabolism, and Disease. Cell 168, 960-976 (2017).

10. Laplante, M. \& Sabatini, D. M. mTOR signaling at a glance. J. Cell Sci. 122, 3589-3594 (2009).

11. Laplante, M. \& Sabatini, D. M. mTOR signaling. Cold Spring Harb. Perspect. Biol. 4, (2012).

12. Jefferies, H. B. J., Reinhard, C., Kozma, S. C. \& Thomas, G. Rapamycin selectively 
represses translation of the 'polypyrimidine tract' mRNA family. Proc. Natl. Acad. Sci. U. S. A. 91, 4441-4445 (1994).

13. Avni, D., Biberman, Y. \& Meyuhas, O. The 5' terminal oligopyrimidine tract confers translational control on TOP mRNAs in a cell type-and sequence context-dependent manner. Nucleic Acids Research 25, (Oxford University Press, 1997).

14. Meyuhas, O. Synthesis of the translational apparatus is regulated at the translational level. European Journal of Biochemistry 267, 6321-6330 (2000).

15. Gentilella, A. \& Thomas, G. Cancer biology: The director's cut. Nature 485, 50-51 (2012).

16. Pasacantilli, I., Frisone, P., De Paola, E., Fidaleo, M. \& Paronetto, M. P. hnRNPM guides an alternative splicing program in response to inhibition of the $\mathrm{PI} 3 \mathrm{~K} / \mathrm{AKT} / \mathrm{mTOR}$ pathway in Ewing sarcoma cells | Nucleic Acids Research | Oxford Academic. Nucleic Acids Res. 45, 12270-12284 (2017).

17. Lee, G. et al. Post-transcriptional Regulation of De Novo Lipogenesis by mTORC1S6K1-SRPK2 Signaling. Cell 171, 1545-1558.e18 (2017).

18. Csibi, A. et al. The mTORC1 pathway stimulates glutamine metabolism and cell proliferation by repressing SIRT4. Cell 153, 840-854 (2013).

19. Chang, J. W. et al. MRNA 3'-UTR shortening is a molecular signature of mTORC1 activation. Nat. Commun. 6, 1-9 (2015).

20. Chang, J.-W. et al. An Integrative Model for Alternative Polyadenylation, IntMAP, Delineates mTOR-modulated Endoplasmic Reticulum Stress Response - PubMed. Nucleic Acids Res. 46, 5996-6008 (2018).

21. Chang, J.-W. et al. mTOR-regulated U2af1 tandem exon splicing specifies transcriptome 
features for translational control. Nucleic Acids Res. (2019). doi:10.1093/nar/gkz761

22. Fahmi, N. A. et al. AS-Quant: Detection and Visualization of Alternative Splicing Events with RNA-seq Data. bioRxiv 2020.02.15.950287 (2020). doi:10.1101/2020.02.15.950287

23. S, H., JO, M.-H., R, V., SP, K. \& Y, H. The Long and the Short of It: The MDM4 Tail So Far. J. Mol. Cell Biol. 11, (2019).

24. Philippe, L., Vasseur, J.-J., Oise Debart, F. \& Thoreen, C. C. La-related protein 1 (LARP1) repression of TOP mRNA translation is mediated through its cap-binding domain and controlled by an adjacent regulatory region. Nucleic Acids Res. 46, 14571469 (2017).

25. Nandagopal, N. \& Roux, P. P. Regulation of global and specific mRNA translation by the mTOR signaling pathway. Translation 3, e983402 (2015).

26. Rubio, C. A. et al. Transcriptome-wide characterization of the eIF4A signature highlights plasticity in translation regulation. Genome Biol. 15, 476 (2014).

27. Hinnebusch, A. G., Ivanov, I. P. \& Sonenberg, N. Translational control by 5'-untranslated regions of eukaryotic mRNAs. Science 352, 1413-1416 (2016).

28. Araujo, P. R. et al. Before It Gets Started: Regulating Translation at the 5' UTR PubMed. Comp Funct Genomics (2012).

29. Mignone, F., Gissi, C., Liuni, S. \& Pesole, G. Untranslated regions of mRNAs. Genome Biology 3, (2002).

30. Zhou, Z. et al. Comprehensive Analysis of Lysine Acetylome Reveals a Site-Specific Pattern in Rapamycin-Induced Autophagy. J. Proteome Res. 18, 865-877 (2019).

31. Zhao, J., Zhai, B., Gygi, S. P. \& Goldberg, A. L. MTOR inhibition activates overall protein degradation by the ubiquitin proteasome system as well as by autophagy. Proc. 
Natl. Acad. Sci. U. S. A. 112, 15790-15797 (2015).

32. Lin, H., Su, X. \& He, B. Protein lysine acylation and cysteine succination by intermediates of energy metabolism. ACS Chemical Biology 7, 947-960 (2012).

33. Zoncu, R., Efeyan, A. \& Sabatini, D. M. MTOR: From growth signal integration to cancer, diabetes and ageing. Nature Reviews Molecular Cell Biology 12, 21-35 (2011).

34. Pereira, J. M. et al. Infection Reveals a Modification of SIRT2 Critical for Chromatin Association. Cell Rep. 23, 1124-1137 (2018).

35. Rack, J. G. M., Vanlinden, M. R., Lutter, T., Aasland, R. \& Ziegler, M. Constitutive nuclear localization of an alternatively spliced sirtuin-2 isoform. J. Mol. Biol. 426, 16771691 (2014).

36. Chang, J. W. et al. MRNA 3'-UTR shortening is a molecular signature of mTORC1 activation. Nat. Commun. 6, 1-9 (2015).

37. Änkö, M. L. et al. The RNA-binding landscapes of two SR proteins reveal unique functions and binding to diverse RNA classes. Genome Biol. 13, (2012).

38. Paz, I., Kosti, I., Ares, M., Cline, M. \& Mandel-Gutfreund, Y. RBPmap: a web server for mapping binding sites of RNA-binding proteins. Nucleic Acids Res. 42, 361-367 (2014).

39. Massey, F. J. The Kolmogorov-Smirnov Test for Goodness of Fit. J. Am. Stat. Assoc. 46, 68-78 (1951).

40. Kim, D. et al. TopHat2: Accurate alignment of transcriptomes in the presence of insertions, deletions and gene fusions. Genome Biol. 14, (2013).

41. Bray, N. L., Pimentel, H., Melsted, P. \& Pachter, L. Near-optimal probabilistic RNA-seq quantification. Nat. Biotechnol. 34, 525-527 (2016).

42. Reference Sequence (RefSeq) Database at NCBI: Current Status, Taxonomic Expansion, 
and Functional Annotation - PubMed. Available at:

https://pubmed.ncbi.nlm.nih.gov/26553804/. (Accessed: 28th June 2020)

43. The Pfam Protein Families Database - PubMed. Available at:

https://pubmed.ncbi.nlm.nih.gov/22127870/. (Accessed: 28th June 2020)

44. The InterPro Protein Families Database: The Classification Resource After 15 Years -

PubMed. Available at: https://pubmed.ncbi.nlm.nih.gov/25428371/. (Accessed: 28th June 2020)

45. The KEGG Databases at GenomeNet - PubMed. Available at:

https://pubmed.ncbi.nlm.nih.gov/11752249/. (Accessed: 28th June 2020)

46. g:Profiler: a web server for functional enrichment analysis and conversions of gene lists (2019 update) | Nucleic Acids Research | Oxford Academic. Available at:

https://academic.oup.com/nar/article/47/W1/W191/5486750. (Accessed: 28th June 2020) 


\section{Methods}

\section{Cell Lines}

WT, Tsc1-/- MEFs, HEK293, MCF7, and MDA-MB-361 cells were cultured in Dulbecco’s Modified Eagle Media (DMEM) (Gibco), in supplements with 10\% FBS (Gibco), $100 \mathrm{ug} / \mathrm{ml}$ streptomycin, and $100 \mathrm{U} / \mathrm{ml}$ penicillin. All cell lines were cultured at $37^{\circ} \mathrm{C}$ with $5 \% \mathrm{CO}$.

\section{Plasmids}

cDNAs of mouse Sirt2 long form (NM_022432), Sirt2 short form (NM_001122765), mouse mdm2 long form (NM_010786.4), mdm2 short form (NM_001288586.2) were cloned into p3xFlag-CMV-14 plasmid.

\section{Real-time PCR (RT-PCR) and Real-time quantitative PCR (RT-PCR) analysis}

Total RNAs were isolated using the Trizol method recommended by manufacturer's protocol. cDNAs were made by reverse transcription using Oligo-d(T) and superscript III (Thermo Fisher Scientific). SYBR Green fluorescence was used for quantitative real-time PCR reactions. The relative expression between groups was measured using the $\Delta \Delta \mathrm{Ct}$ method. The list of primers used for RT-PCR and qRT-PCR is:

mouse Camkk2 RT-PCR forward: 5'-GGGAACCCGTTCGAAGGTAG-3'; mouse Camkk2 RTPCR reverse: 5'-GCAGGGACCACCTTTCACAA-3'; mouse Ranbp3 RT-PCR forward: 5'AAGCCTGCCGTCGCACCGTCTGTCT; mouse Ranbp3 RT-PCR reverse: 5' CTTCTCCGGCTTCGGGACTGGAGC-3'; mouse Mdm4 RT-PCR forward: 5'GCTAAGAAAGAATCTTGTTACATCAGC-3'; mouse Mdm4 RT-PCR reverse: 5'- 
ATGtCGTGAGGtAGGCAGTGTGTGA-3'; mouse Syce2 RT-PCR forward: 5'AGCATCGGCAGAGTGAGAAC-3'; mouse Syce2 RT-PCR reverse: 5'CCGTTTCCACAGTTTGGCAG-3'; mouse Itch RT-PCR forward: 5'TTCACAGTGGCCTTCTGGAAACAACG-3'; mouse Itch RT-PCR reverse: 5'GGAATCAAGCTGTGGTCCACTGTCAGA-3'; mouse Cenpi RT-PCR forward: 5'AgAGgCtGtGTGCTCGCCGAGGT-3'; mouse Cenpi RT-PCR reverse: 5'TGAAAGCAGATGACTGGCCTACAGTAG-3'; mouse Gabpb2 forward: 5'GAGAACCAACTCCTGCGAGTTGTC-3'; mouse Gabpb2 reverse: 5'TCCCCAAGTCCACCAGAGACATCTTA-3'; human MDM4 forward: 5'CGTCAGAGCTTCTCCGTGAAAGAC-3'; human MDM4 reverse: 5'CTCTAGAATGTATGCATTTATGCTC-3'; mouse Eef2 RT-PCR forward: 5'AGTGTCCTGAGCAAGTGGTG-3'; mouse Eef2 RT-PCR reverse: 5'AGATCAGCGGTGAAGCCAAA-3'; mouse Hsp90ab1 RT-PCR forward: 5'CAGAAGGCTGAGGCAGACAA-3'; mouse Hsp90ab1 RT-PCR reverse: 5'AATCATGCGGTAGATGCGGT-3'; mouse Anksl RT-PCR forward: 5'GCTGACTCGAAAGGCTGCTACC-3'; mouse Anksl RT-PCR reverse: 5'TCCAGGGGCGTTTCAAACTTGTTG-3'; mouse Ect2 RT-PCR forward: 5'GAAATGCCGCAGGTTGAAGCAAG-3'; mouse Ect2 RT-PCR reverse: 5'ACTGGTGGCCCAACAATCCTACA-3'; mouse Adgrl2 RT-PCR forward: 5'TCCTCTGTGAGGCTGATGGAAC-3'; mouse Adgrl2 RT-PCR reverse 5'CAACAACATTGTGGCTGTGTGCG-3'.

\section{siRNA knockdown and antibodies}


Cells were transfected with siRNAs synthesized by Integrated DNA Technologies (IDT) using RNAiMax Transfection reagents (Thermo Fisher Scientific) recommended by the manufacturer's protocol. siRNA targeting mouse Srsf3: 5'-CGUGAUAUCAAGAAUUGU-3'. Antibodies used for western blot analysis include: anti-Flag (No. F3165, Sigma Aldrich), anti-S6 (No. 2317, Cell Signaling), Secondary antibody against mouse: goat-anti-mouse IgG-HRP (No. sc-2005, Santa Cruz Biotechnology), Secondary antibody against rabbit: goat-anti-rabbit IgG-HRP (No. sc-2004, Santa Cruz Biotechnology).

\section{Polysome isolation and analysis}

Polysomes from mock-treated and Torin1-treated Tsc1/- MEF cell lysates were isolated using a sucrose gradient ${ }^{40}$. Cells were lysed in lysis buffer containing $20 \mathrm{mM}$ Tris $\mathrm{pH}=7.4,150 \mathrm{mM}$ $\mathrm{NaCl}, 5 \mathrm{mM} \mathrm{MgCl} 2,1 \mathrm{mM}$ dithiothreitol, $100 \mathrm{mb} / \mathrm{ml}$ cycloheximide, $1 \%$ TritonX-100. Cytoplasmic extracts were loaded onto the sucrose gradient of $5 \%$ to $45 \%$ followed by ultracentrifugation of $36,000 \mathrm{rpm}$ for 2 hours at $4^{\circ} \mathrm{C}$. Eleven fractions were collected in total. Total RNAs were isolated using the Trizol method stated above. Ten percent $(\mathrm{v} / \mathrm{v})$ of the total RNAs from each fraction was used for cDNA synthesis for RT-PCR analysis. The total intensity was determined from the band intensity of the transcript isoform of each fraction combined and set as $100 \%$. The relative ratio of the band intensity from each fraction over the total intensity was calculated and was used to generate the cumulative plots. Data presented shows the comparison of the two AS transcript in each condition (mock and Torin 1) For each cumulative plot, the x-axis denotes the fraction number, whereas the $\mathrm{y}$-axis is the cumulative probability. To check the statistical difference between the two AS transcript isoforms in each condition, the Kolmogorov-Smirnov ${ }^{39}$ was applied and the $p$-value was reported in each plot. 


\section{mRNA stability}

Cells were treated with $1 \mathrm{ug} / \mathrm{ml}$ actinomycin D to inhibit transcription and were harvested at different time points in the course of 12 hours. Total RNAs were obtained using the Trizol method stated above.

\section{Protein stability}

Cells were treated with $30 \mathrm{ug} / \mathrm{ml}$ cycloheximide to inhibit protein synthesis and were harvested at different time points. Cells were then lysed and lysates were run on SDS PAGE gels for western blot analysis.

\section{RNA-seq Data Analyses}

To evaluate the transcriptome features under mTOR-hyperactivation at single-nucleotide resolution, we performed RNA-Seq analyses of poly(A+) RNAs isolated from WT, $T s c 1^{-/-} \mathrm{MEFs}$, MCF7 and MDA-MB-361 cells. Paired-end reads were aligned to the mouse mm10 reference genome or human hg19 reference genome using TopHat $2^{40}$ with up to two mismatches allowed. Kallisto $^{41}$ was applied to quantify gene expressions with RefSeq annotation ${ }^{42}$.

\section{AS-Quant (Alternative Splicing Quantitation) pipeline}

Alternative splicing analysis was performed using the AS-Quant pipeline ${ }^{22}$. Briefly, the pipeline categorizes the alternative splicing events into cassette exon, mutually exclusive, alternative 5' splice site, and alternative 3' splice site. Reads (n) from these alternatively spliced exons were measured and compared to reads $(\mathrm{N})$ coverage of the rest of the transcripts. A $2 \times 2$ Chi-Square test 
was performed to determine the statistical significance for each alternative splicing event using the ratio of $\mathrm{n} / \mathrm{N}$. Events with a $\mathrm{p}$-value $<0.1$ and a ratio difference $>0.1$ were considered real alternative splicing exons.

\section{Pfam domain analysis}

Pfam-Scan was used to search Pfam databases for the matched Pfam domains on each transcript with 1e-5 e-value cutoff ${ }^{43}$. Only the Pfam domains which were overlapped with skipped exon(s) were considered for the further analysis. The InterPro Protein Families Database ${ }^{44}$ provided the information to link the Pfam domains to the Gene Ontology terms. The LinkDB under the GenomeNet Database ${ }^{45}$ provided the information to link the Pfam domains to the KEGG pathways.

\section{Quantification and Statistical Analysis}

Signal intensity from gel electrophoresis or western blots was quantified using the ImageStudio software. Statistical analysis was performed by Two-tailed Student's test unless stated otherwise.

\section{Data Availability}

The accession numbers for the RNA-seq data are SRP056624. 


\section{Supplemental Figures}

A

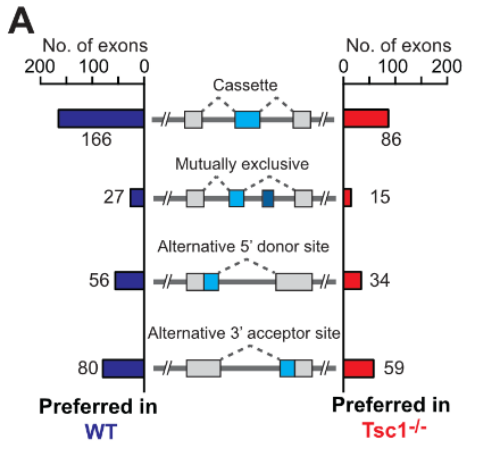

B

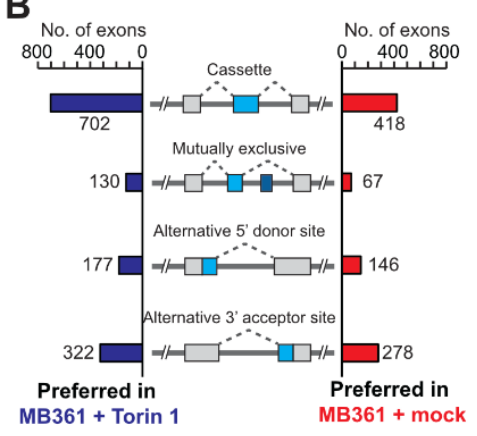

C
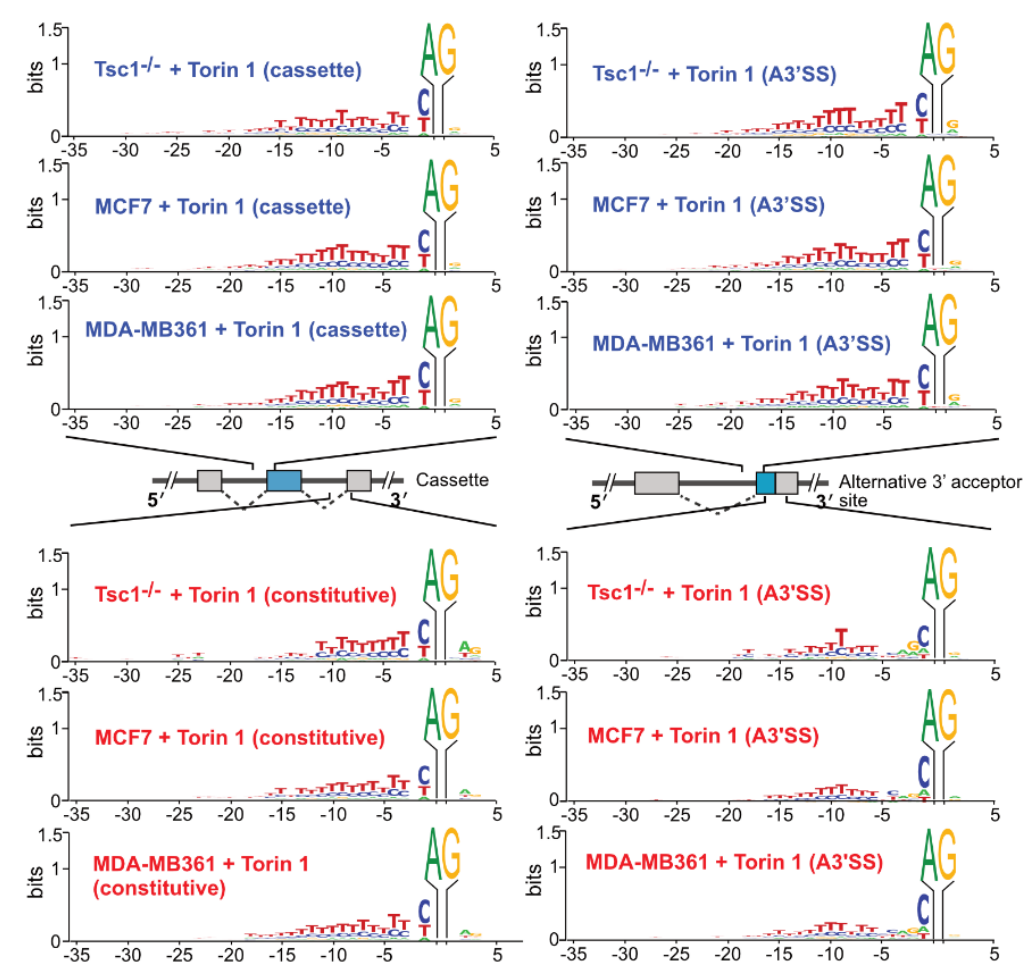

D

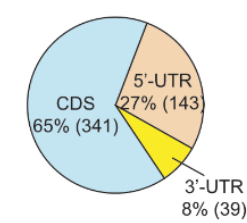

WT vs. Tsc1\%

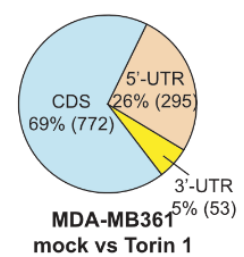

Supplemental Fig. 1. Cases of alternative exon inclusion when comparing A) WT vs. Tsc1 ${ }^{-/}$ MEFs and B) MDA-MB-361 mock vs Torin 1 treatment. C) The frequency of sequence preference of the 3 '-splice site at AS exon compared to constitutively spliced exons for Tsc1 ${ }^{-/}$, MCF7, and MDA-MB-361 mock- and Torin 1- treated cells. X-axis represents the nucleotide position relative to the 3 ' $\mathrm{AG}$ dinucleotide and $\mathrm{Y}$-axis represents the certainty of the nucleotide. 
bioRxiv preprint doi: https://doi.org/10.1101/2020.02.27.967737; this version posted June 29, 2020. The copyright holder for this preprint (which was not certified by peer review) is the author/funder. All rights reserved. No reuse allowed without permission.

D) Distribution of mTOR-regulated alternative splicing (AS) events in different regions of the mRNA. 
bioRxiv preprint doi: https://doi.org/10.1101/2020.02.27.967737; this version posted June 29, 2020. The copyright holder for this preprint (which was not certified by peer review) is the author/funder. All rights reserved. No reuse allowed without permission.

A
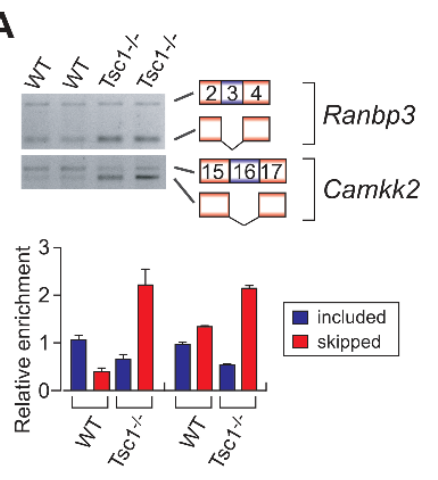

$\mathbf{F}$

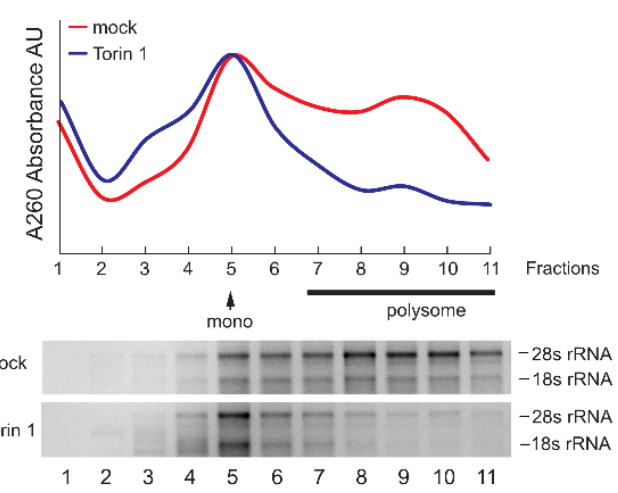

B

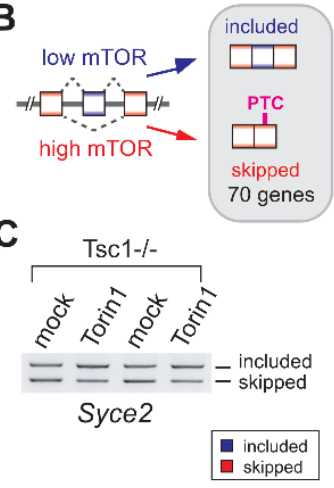

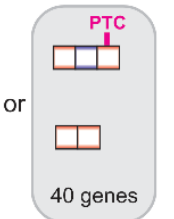

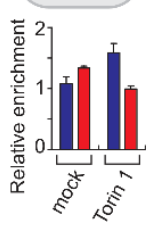

D

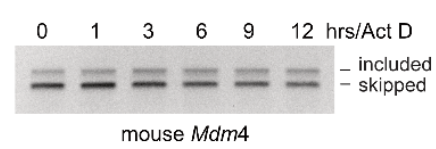

E

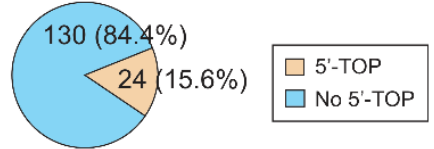

5'UTR AS

WT vs. Tsc1\%

G

Eef2

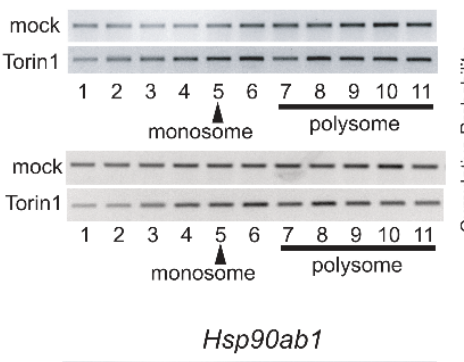

mock - - - - - - - Torin1 - - - - - - - - $\begin{array}{lllllllllll}1 & 2 & 3 & 4 & 5 & 6 & 7 & 8 & 9 & 10 & 11\end{array}$ mock - - - - - - - Torin1 - - - - - - - - -

$\begin{array}{lllllllllll}1 & 2 & 3 & 4 & 5 & 6 & 7 & 8 & 9 & 10 & 11\end{array}$

Hsp90ab1

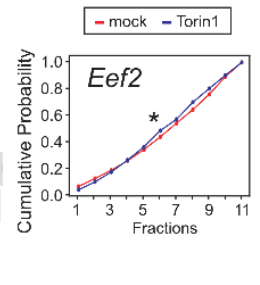

\begin{tabular}{c} 
mock \\
Torin1 \\
\hline
\end{tabular} mock - - - - - - - Torin1 - - - - - - - -

$\begin{array}{lllllllllll}1 & 2 & 3 & 4 & 5 & 6 & 7 & 8 & 9 & 10 & 11 \\ \text { monosóme } & & & & \text { polysome }\end{array}$

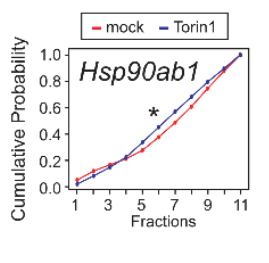

H
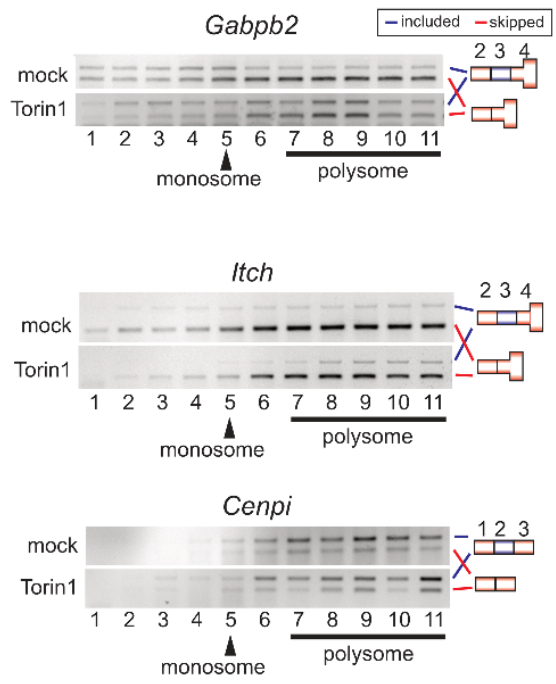
Supplemental Fig. 2 A) Semi-quantitative RT-PCR validation of mTOR-regulated AS events. (top panel) RT-PCR and analytical gel electrophoresis were conducted in WT and Tsc1-- MEFs. Two technical repeats were done. (bottom panel) Quantitation of gel images was done using ImageStudioLite. B) Schematic diagram illustrating the transition between coding and noncoding transcript caused by AS of cryptic exons. The number of genes showing coding to noncoding or vice versa by mTOR-driven AS events is shown. C) Semi-quantitative RT-PCR validation of coding-to-noncoding isoform switches. Syce2 AS event was analyzed in Tsc1 $1^{-/}$MEFs in the presence or absence of Torin 1 treatment $(50 \mathrm{uM})$. The relative quantification of the coding and noncoding transcripts was done using densitometry analysis. D) Technical repeat of stability of $m d m 4$ isoform transcripts. Semi-quantitative RT-PCR analysis was performed using Tsc $1^{-/-}$ MEFs treated with $1 \mathrm{ug} / \mathrm{ml}$ actinomycin D for indicated time points. E) Proportion of 5'-UTR AS events containing the 5'TOP motif in WT vs Tsc1/-- MEFs. F) A260 absorbance plot and denatured RNA agarose electrophoresis of total RNAs from polysome profiling sucrose fractions in mock- and Torin 1- treated Tsc $1^{-/-}$MEFs. G) Polysome analyses of 5'-TOP containing transcripts eef2 and hsp90ab1. Semi-quantitative RT-PCR and analytical gel electrophoresis were performed on the cytoplasmic fractions of mock- and Torin 1-treated Tsc1 ${ }^{-/-}$MEFs. The monosome and polysome fractions are indicated. The cumulative distribution of the transcript in each fraction is plotted against the summation of that isoform in all 11 fractions. The $\mathrm{x}$-axis indicates the fraction number and $y$-axis indicates the cumulative probability. H) Technical repeat of polysome analyses of mTOR-regulated 5'-UTR AS transcripts. 


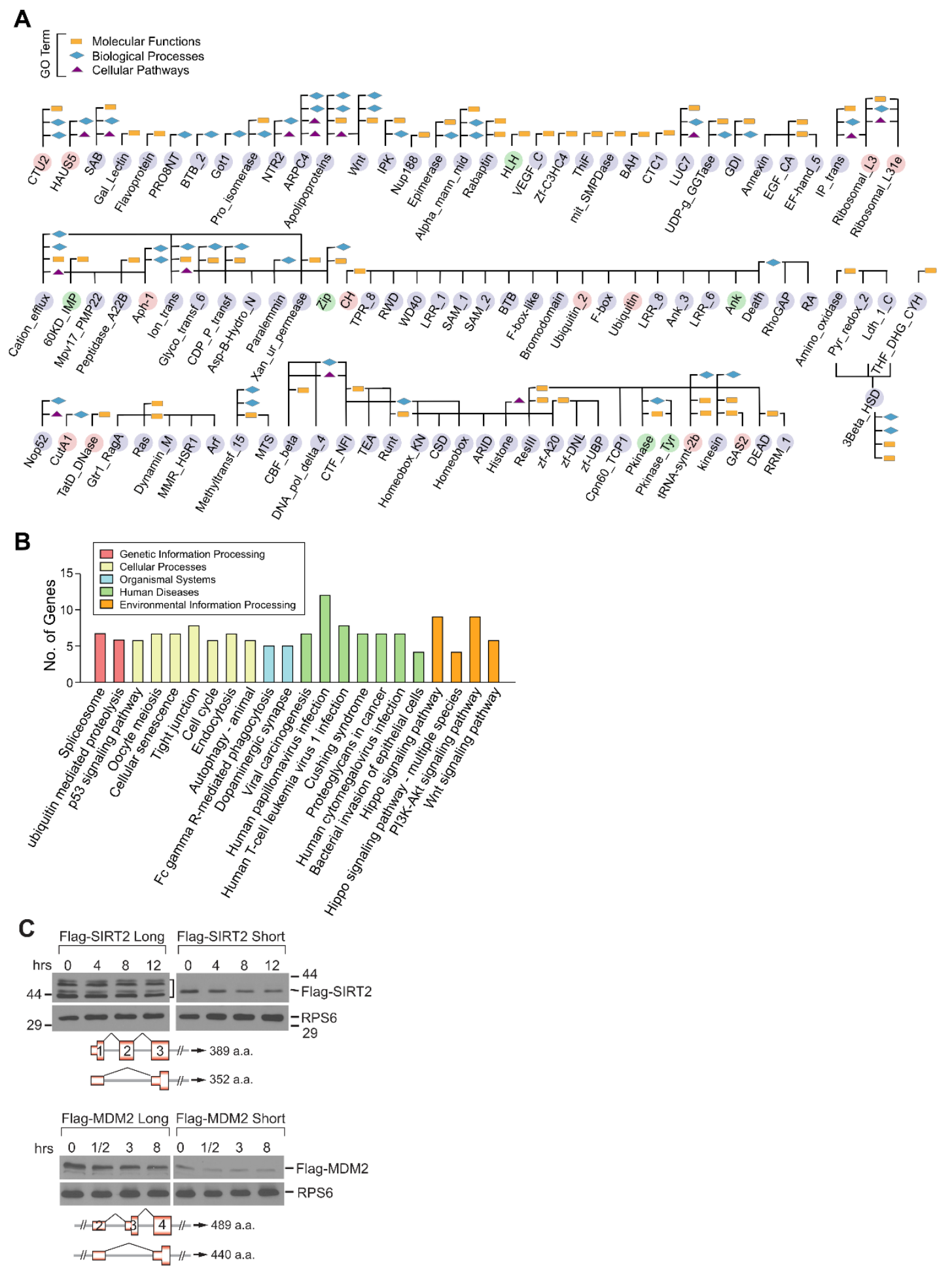


Supplemental Fig. 3 A) All of the impacted Pfam domains and their associated GO terms by mTOR-driven AS events. Bioinformatics analysis of the functional Pfam domains disrupted by AS events in the coding region of mRNA was done. Pink: Pfam domains gained in hyperactivated mTOR. Light purple: Pfam domains lost in hyper-activated mTOR. Green: Pfam domains present in both low and high mTOR environments. Rectangle: Molecular Functions; Diamond: Biological Processes; Triangle: Cellular Pathways. Each of affected Pfam domains associated with a GO term is indicated by a linkage to rectangle, diamond, and/or triangle. B) Affected KEGG pathways by mTOR-driven AS events determined using g:Profiler ${ }^{44}$. The KEGG pathways are grouped into 5 categories, shown by the legend. C) Technical repeat of protein isoform stability by western blotting. Flag-tagged SIRT2 and MDM2 protein isoforms were transiently expressed in HEK293 and the differences in their stabilities was monitored in the presence of cycloheximide $(30 \mathrm{ug} / \mathrm{ml})$ for indicated time points. Ribosomal protein S6 (RPS6) was loaded as a control. The protein level was quantified using densitometry and normalized to the S6 level. 
A

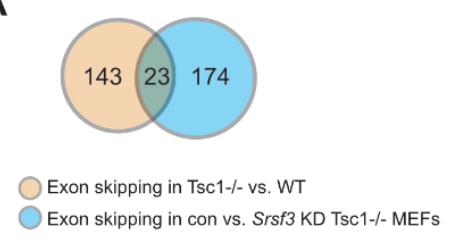

B
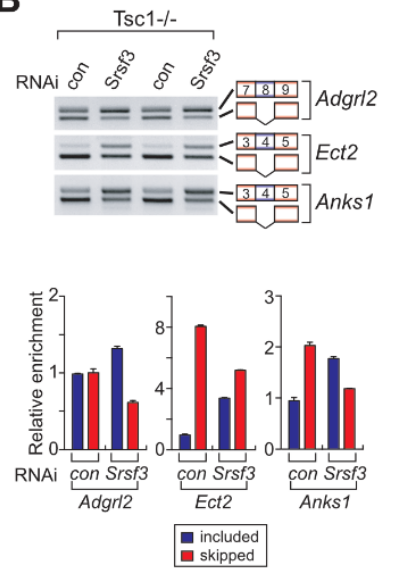
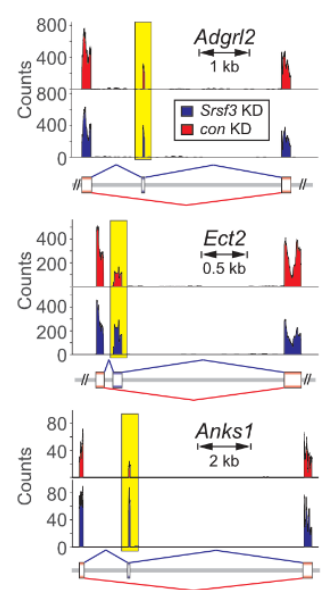

Supplemental Figure 4. A) Venn diagram illustrating the overlap of skipped exons in WT vs. $\mathrm{Tsc}^{-/-} \mathrm{MEF}$ dataset and control vs. Srsf3 knockdown in Tsc1 ${ }^{-/-}$MEFs dataset. B) Semiquantitative RT-PCR validation of $\operatorname{Srs} 33$ knockdown RNA-seq experiment. Alternative splicing of Anks1, Ect2, and Adgrl2 were tested in control knockdown and $S r s f 3$ knockdown Tsc1/MEFs. Tsc1 ${ }^{-/-}$MEFs were transfected with scrambled or Srsf3 siRNAs for 48hrs, and total RNAs were harvested. mRNAs were reverse transcribed into cDNA, which served as a template for RT-PCR. 